\title{
NOTAS SOBRE LA CONFIGURACIÓN DE LAS UNIONES DE HECHO EN ROMA
}

\author{
WALENKA ARÉVALO CABALLERO \\ Universidad de Alicante
}

Las uniones de hecho se caracterizan en la actualidad por ser uno de los temas más candentes que se plantea a los estudiosos del Derecho, porque si bien, hasta ahora y durante muchos siglos fue el matrimonio la institución en la que descansaba la seguridad de la familia y la estabilidad de la nación ${ }^{1}$, hoy día se están constituyendo nuevos tipos de convivencia familiar de los que se derivan innumerables problemas jurídicos a los que se debe procurar correcta solución.

Cuando se alude a parejas de hecho, tanto en Roma como en época actual, se hace referencia a uniones estables y no de mera relación sexual que deben cumplir determinados requisitos, recogidos por la jurisprudencia, en los que se evita la voluntad de los convivientes de contraer matrimonio conforme a las normas del Ordenamiento jurídico.

Ahora bien, antes de abordar el tema de las uniones de hecho en Roma o por citarlo en terminología latina -concubinatus-, se debe exponer, aunque sea sucintamente las líneas generales del matrimonio romano².

1. TORRENT, A.: "Uniones de hecho, nueva tipología de la familia», en Libro homenaje al profesor Manuel Albaladejo Garcia, Murcia, Universidad de Murcia, 2004, pp. 4783 ss.

2. Para conocer más sobre el tema vid. entre otros: BRINI, G.: Matrimonio e divorzio nel diritto romano, Bologna, Nicola Zanicchelli, 3 vols. 1886-1889; DI MARZO, S.: Lezioni sul matrimonio romano, reed. Palermo, L'Erma di Bretchneider, 1972; VOLTERRA, E.: La conception du mariage d'aprés les juristes romains, Padova, La Garangola, 1940; ORESTANO, R.: «La estruttura giuridica del matrimonio romano dal diritto clásico al diritto giustinianeo», BIDR, 55-56 (1952), pp. 185 ss.; LAURIA, M.: Matrimonio e dote, Napoli, L'Arte Tipográfica, 1952; Bonfante, P.: Corso di Diritto Romano, Diritto di Familia, vol. I., Milano, Giuffré, 1963, pp. 255 ss.; RoBledA, O.: El matrimonio en Derecho Romano, Roma, Università Gregoriana, 1970; DAZA, J.: Nuptiae et matrimonium, Estudios Álvarez Suárez, Madrid, 1978, pp. 57 ss.; VolterRA, E.: Instituciones de Derecho Privado Romano, Madrid, 1991 (trad. español por J. Daza), pp. 635 ss.; TorRent, A.: Manual de Derecho privado Romano, Zaragoza, Edisofer, 1995, pp. 525 ss.; PANERO, R.: Derecho Romano, Valencia, Tirant lo Blanc, 2000 , pp. 289 ss. 
Originariamente el matrimonio en Roma fue una institución que no presentaba vínculos jurídicos sino primordialmente connotaciones sociales, éticas y religiosas ${ }^{3}$. No obstante, desde época arcaica, se planteó la cuestión de establecer los requisitos necesarios para que la unión entre un hombre y una mujer, ciudadanos romanos, pudiera lograr determinadas consecuencias jurídicas. A este respecto, es necesario indicar que entre los efectos jurídicos más significativos que se establecieron para dar estabilidad a esta relación se encontraban, por una parte la determinación de la filiación de los hijos nacidos de esa unión y por otra la concesión a padres e hijos de derechos y deberes recíprocos; pero sobre todo y como efecto de mayor trascendencia, la atribución al hijo de la condición de ciudadano romano que ostentaban los padres ${ }^{4}$.

Con referencia al status civitatis de los hijos, se determinó que el nacido de iustum matrimonium ${ }^{5}$ adquiría la condición que ostentaba el padre en el momento de la concepción, por lo tanto, si el padre era ciudadano romano casado en iustas nupcias con ciudadana romana o con latina o peregrina con ius conubii ${ }^{6}$, el hijo nacía ciudadano romano, al establecerse que el filius siguiese la condición que ostentaba el padre ${ }^{7}$.

Ahora bien, si la madre era ciudadana romana y estaba casada con un latino o un peregrino con ius conubii, los hijos nacerían con el status de latinos o peregrinos, puesto que, como hemos señalado, debían seguir la condición del padre.

Sin embargo, hay que advertir que sobre el reconocimiento del status civitatis se establecía un derecho particular para las mujeres, aunque desgraciadamente no permaneció durante mucho tiempo, pues en principio, al nacido de una ciudadana romana y un latino o peregrino sin ius conubii que constituían un

3. Torrent, A.: Manual...,op. cit., p. 525.

4. Sobre el tema vid. POLO ARÉVALO, E.M.: «Influencia del matrimonio y condición ciudadana de los padres en el status civitatis y familiae de los hijos», en El Derecho de familia de Roma al Derecho actual, Huelva, Asociación Iberoamericana de Derecho Romano, 2004, pp. 585 ss.

5. Los requisitos del matrimonio iustum se establecen en los Tituli ex corpore Ulpiani. 5, 2 Iustum matrimonium est, si inter eos, qui nuptias contrahunt, conubium sit, et tam masculus pubes quam femina potens sit, et utrique consentiant, si sui iuris sunt, aut etiam parentes eorum, si in potestate sunt, esto es, existe justo matrimonio si entre los que contraen nupcias existe conubium, el varón es púber, la mujer núbil y ambos consienten si son sui iuris, o en el caso de que esten in potestate den su consentimiento también los padres.

6. El conubiun era la capacidad jurídica para contraer matrimonio. Sobre la materia, vid. entre otros: VolterRA, E.: «La nozione giuridica del conubium», Studi Albertario, II (1959), pp. 374 ss.; DE VISSCHER, F.: "Conubium et civitas», IURA, II (1951), pp. 140 ss.; GAUDEMET, J.: "Iustum matrimonium», RIDA, III (1956), pp. 227 ss.

7. Gayo expone que los concebidos en justas nupcias no solo entraban bajo la potestad del padre sino que adquirían la condición de ciudadanos romanos: GAI 1, 56. Iustas autem nuptias contraxisse liberosque iis procreatos in potestate habere cives Romani ita intelleguntur, si cives Romanas uxores duxerint vel etiam Latinas peregrinasve, cum quibus conubium habeant: Cum enim conubium id efficiat, ut liberi patris condicionem sequantur, evenit, ut non solum cives Romani fiant, sed et in potestate patris sint. 57. Unde et veteranis quibusdam concedi solet principalibus constitutionibus conubium cum his Latinis peregrinisve, quas primas post missionem uxores duxerint; et qui ex eo matrimonio nascuntur, et cives Romani et in potestatem parentum fiunt. 
matrimonium iniustum ${ }^{8}$, se le concedía la ciudadanía romana si la madre la poseía en el momento del nacimiento de su hijo, con independencia de que la hubiese ostentado o no con anterioridad.

Este privilegio fue modificado por una lex Minicia del año 90 a.C., estableciendo que los hijos de las uniones impropias siguieran el status civitatis del padre, por ello, a las mujeres que quisieran concebir hijos ciudadanos romanos, no les quedaba otra opción que casarse con cives romanos.

Analizando el matrimonio en Roma, se puede constatar que era una situación de hecho más que de derecho y por ello, no se encuentra en las fuentes ninguna definición jurídica del matrimonio; al respecto hay que señalar que los juristas romanos no plantearon una noción clara de la institución sino que establecieron meras aproximaciones que destacaban el hecho de la unidad de vida entre dos seres de distinto sexo, como se constata en las Instituciones de Justiniano 1,9,1, donde se establece que el matrimonio era la unión de un hombre y una mujer dirigida a una unidad de vida; concepto que perfila más una realidad sociológica que un instituto jurídico?.

I. 1,9,1 -Nuptiae autem sive matrimonium est viri et mulieris coniunctio, individuam consuetudinem vitae continens.

Se vuelve a acentuar el carácter de comunidad de vida en la célebre definición de Modestino, contenida en D. 23, 2, 1, en la que se destacan tres aspectos en el concepto de matrimonio, al manifestar que, eran nupcias la unión de un hombre y mujer, el consorcio de toda la vida y la comunicación de derecho divino y humano.

D. 23. 2. 1 -Modestinus 1 reg. -Nuptiae sunt coniunctio maris et feminae et consortium omnis vitae, divini et humani iuris communicatio.

El jurista, en ningún momento se refiere a las consecuencias jurídicas que pudiese llevar consigo esa unión, lo que pone de manifiesto, que el matrimonio en Roma era esencialmente, como ya se ha manifestado, una situación de hecho basado en la praxis de las relaciones sociales, sin tener en cuenta vínculos jurídicos.

No obstante, se podría establecer una noción del matrimonio en época clásica, siguiendo a Bonfante ${ }^{10}$ manifestando que existía matrimonio cuando se establecía la unión de un hombre y una mujer libres, que ostentaban conubium, tenían la edad prescrita por la ley -hombre púber y mujer núbil- y creaban una relación conyugal con la voluntad efectiva y continua de estar unidos en matrimonio.

8. VolterRA, E.: «Iniustum matrimonio», Scritti Giuridici, III (1991), pp. 441 ss. manifiesta que al matrimonio iniustum le faltaba el conubium, requisito esencial para poder contraer matrimonio conforme a las reglas del Ordenamiento jurídico romano, no obstante, no podía considerársele nulo sino que de él no se derivaban los efectos jurídicos propios del matrimonio legítimo.

9. TORRENT, A.: Manual..., op. cit., p. 525.

10. BONFANTE, P.: Corso...,op. cit., p. 258. 
Ahora bien, si el fundamento del matrimonio romano descansaba en el consensus o mero consentimiento de ambos cónyuges si eran sui iuris, expresado sin formalidad alguna y en el caso de que alguno de los esposos estuviera sometido a potestad también era necesario que prestara el consentimiento su pater; no era menos importante la coniunctio o convivencia conyugal de los contrayentes.

Por lo tanto, el matrimonio romano en época clásica comportaba dos elementos, primero el consensus, que se plasmaba en la affectio maritalis, o intención reciproca de los contrayentes de tenerse por marido y mujer; sin embargo, no bastaba con que se conviniera el consensus al inicio del matrimonio sino que debería estar presente todo el tiempo que durase el mismo, por ello, si se perdía la affectio maritalis, se disolvía el matrimonio sin más causa. El segundo se plasmaba en la coniunctio, que no comportaba la convivencia efectiva de los cónyuges sino que la mujer fuese conducida a la casa del marido -deductio in domus mariti- como expone Pomponio en D. 23, 2, $5^{11}$, y en el honor matrimonii por el que la mujer adquiría la misma condición social que el marido, además de ser considerada materfamilias.

Asimismo, para que el matrimonio se considerase iustum, y pudiese obtener los derechos que a tal unión le confería el Ordenamiento jurídico, requería unas condiciones de validez que pueden concretarse en los siguientes requisitos: en primer lugar, capacidad jurídica -conubium- esto es, la facultad conforme al ius civile de constituir entre ellos justo matrimonio; esta aptitud, que en principio fue reservada a los patricios, posteriormente, fue concedida a los plebeyos por la Lex Canuleia. Sin embargo, fuera de la esfera de los ciudadanos romanos, el ius conubi sólo pudo atribuirse a algunos latinos y peregrinos por concesión especial. En segundo lugar, capacidad natural, lo que implicaba que ambos fueran púberes, esto es, que fueran mayores de edad. En tercer lugar, hay que indicar que el matrimonio clásico era monogámico, lo que excluía de la institución a personas unidas por una relación conyugal anterior y exogámico -que no existiese entre los cónyuges parentela consanguínea, de adopción o afinidad-.

La concepción del matrimonio cambiará totalmente en época postclásica, puesto que la legislación imperial abolió la necesidad del ius conubii para contraer justo matrimonio y estableció una modificación en la forma de prestar el consentimiento matrimonial, ya que, si bien en época clásica debía ser continuado durante todo el tiempo que permaneciera la relación conyugal, se transformará en inicial por obra de los emperadores cristianos, esto es, que únicamente sería requerido al inicio de la relación; por tanto, el consensus debía interpretarse como la voluntad de los contrayentes de unirse en matrimonio, con independencia de que tal voluntad persistiese o cambiase.

La transformación de la prestación del consentimiento de continuada a inicial, tendrá como consecuencia que el matrimonio ya no se disolverá libremen-

11. D. 23, 2, 5-Pomponius 4 ad sab.-Mulierem absenti per litteras eius vel per nuntium posse nubere placet, si in domum eius deduceretur: eam vero quae abesset ex litteris vel nuntio suo duci a marito non posse: deductione enim opus esse in mariti, non in uxoris domum, quasi in domicilium matrimonii. 
te con la perdida de la affectio maritalis, sino que para poder divorciarse debían concurrir toda una serie de causas tipificadas en la legislación, cuyo efecto era el de mantener vivo el vínculo matrimonial.

Después de haber expuesto en líneas generales los requisitos del matrimonio romano, se analizará el concepto de concubinato o unión de hecho en Roma.

Considerando los requisitos necesarios para contraer matrimonio en época clásica, se puede determinar que las uniones entre personas de distinto sexo que no tuvieran conubium no podían constituir matrimonio iustum, por lo que su convivencia podría ser calificada en la actualidad como unión de hecho. Igualmente, si faltaba el consentimiento de las partes para formar matrimonio -affectio maritalis-, también sería consideraba esa unión concubinatus o unión de hecho.

Por tanto, la doctrina romanística ${ }^{12}$ es conforme al definir el concubinato -unión de hecho-, como la convivencia estable de un hombre y una mujer entre los que no existía conubium ${ }^{13}$, o bien, no tenían la voluntad continua y efectiva de ser recíprocamente marido y mujer.

El concubinato se configuró como una unión de hecho ${ }^{14}$ que se distinguía por su estabilidad de las uniones fugaces de mera relación sexual. Esta institución no fue en Roma ni ilegal ni socialmente reprochable y su fundamento y amplia difusión obedeció, primordialmente, a la legislación de Augusto en materia matrimonial que vino a restringir, notablemente, el número de mujeres con las que podía contraerse iutum matrimonio ${ }^{15}$.

Por un lado, la Lex Iulia et Papia Poppaea prohibía el matrimonio entre personas de distinto rango social para evitar la mezcla de la clase alta-senadores e hijos de senadores- con personas de baja o abyecta condición social, -libertas, artistas, hijas de artistas, meretrices, etc.- y estableció, asimismo, el impedimento para contraer matrimonio entre los gobernadores provinciales y las mujeres de la provincia en la que estaban destinados, prohibición que también se impuso a los militares ${ }^{16}$.

De igual manera, la Lex Iulia et Papia establecía impedimentos matrimoniales a los demás ingenuos, con mujeres libertas, adúlteras, alcahuetas o condenadas en juicio público, entre otras ${ }^{17}$.

12. Entre otros, Bonfante, P.: Corso..., op.cit., p. 315; GAUdemet, J.: «Union libre et marriage dans la Roma Imperiale», IURA, 40 (1989), pp. 1 ss.; VOLTERRA, E.: Instituciones..., op.cit., p. 666; ToRRENT, A.: Manual...,op. cit., p. 537; PANERO, R.: Derecho..., op. cit., p. 305.

13. PANERO ORIA, R.: "Algunas observaciones en torno al concubinato", en El Derecho de familia de Roma al Derecho actual, Huelva, Asociación Iberoamericana de Derecho Romano, 2004, pp. 527 ss., establece que cuando faltaba uno de los presupuestos exigidos para contraer matrimonio, existía affectio maritalis y honor matrimonii, sin embargo, al no poder constituir matrimonio la única posibilidad de mantener una relación estable era el concubinato.

14. PlaSSARD, M.J.: Concubinat romain sous le haut empire, Toulouse-Paris, Dalloz, 1921, p. 17

15. PANERO, R.: Derecho...,op. cit., p. 305.

16. Sobre los impedimentos vid. Astolfr: La lex Iulia et Papia, Padova, 1995.

17. D. 25, 7, 3, pr.-Marcianus 12 inst. -In concubinatu potest esse et aliena liberta et ingenua et maxime ea quae obscuro loco nata est vel quaestum corpore fecit. alioquin si honestae vitae et ingenuam mulierem in concubinatum habere maluerit, sine testatione hoc manifestum faciente non conceditur. sed necesse est ei vel uxorem eam habere vel hoc recusantem stuprum cum ea committere. El jurista se refiere a las mujeres 
Por lo que se refiere a la Lex Iulia de adulteriis, se debe señalar que sancionaba gravemente la unión extraconyugal con mujeres ingenuas y honestas, tipificando los delitos de adulterium y stuprum. Se cometía adulterium cuando la unión se realizaba con mujer casada y stuprum cuando la mujer, ingenua y honesta, era viuda o doncella. Ahora bien, las palabras adulterio y estupro, consignadas en la Lex Iulia, no tenían el significado moderno de violencia e infidelidad conyugal $^{18}$.

Por otra parte, las mujeres de alta condición social, se vieron condicionadas por las leyes augusteas, puesto que no podían casarse con libertos, gladiadores, hijos de gladiadores, etc.

Las prohibiciones de las Leges Iuliae originaron que las uniones constituidas al margen de sus disposiciones fueran consideradas ilegales o contra mandata ${ }^{19}$, puesto que en su virtud se privaba de conubium a los contrayentes, requisito esencial para poder contraer justo matrimonio.

Un punto discutido por la doctrina romanistíca ${ }^{20}$ fue la posibilidad de que las leyes promulgadas por Augusto hubiesen sido las determinantes de la elevación del concubinato de relación social a instituto jurídico, pero se llegó a la conclusión de que las leyes caducarias no establecieron normas que definiesen y regulasen el concubinato y sólo se hacía mención al mismo para declararlo exento de las penas que castigaban el delito de estupro de la Lex Iulia de adulteris y para distinguirlo del matrimonio regulado en la Lex Iulia et Papia ${ }^{21}$.

Sin embargo, hay que precisar que no se redujo el concubinato por el exceso de puritanismo marcado por Augusto sino que su legislación llevó a un resultado contrario, estimulando la propagación de las parejas de hecho al limitar tanto las mujeres como los hombres con los que los ciudadanos romanos podían contraer matrimonio.

El concubinato, expone Bonfante ${ }^{22}$ no era más que una institución de hecho, socialmente tolerada en Roma, pero absolutamente fuera del Derecho; no

con las que se podía vivir en concubinato, aludiendo a la liberta ajena y a la ingenua, no obstante, en caso de que fuera ingenua sólo se podía establecer concubinato con aquella nacida de oscuro linaje o con la que hizo ganancia con su cuerpo, porque si era honesta se cometía stuprum. CASTELLO, C.: In tema di matrimonio e concubinato nel mondo romano, Milano, Giuffré, 1940, p. 189, establece que la mención del concubinato con ingenua es una interpolación justinianea.

18. BONFANTE, P.: Corso..., op. cit., p. 315.

19. Las uniones contra mandata eran aquellas prohibidas por un senadoconsulto o por disposiciones imperiales.

20. Entre los autores que establecen que la elevación del concubinato de relación social a jurídica se debe al emperador Augusto se encuentran, entre otros: MOMMSEM, T.: Romisches Staatsrecht, III, Leipzig, S. Hirzel, 1887, p. 430, n. 2; MEYER, P.M.: Des römische Konkubinat nach den Rechtsquellen und den Inschriften, Leigzig, S. Hirzel, 1895, p. 25; GIDE, P.: Études susr la condition privée de la femme dans le droit ansien et moderne, Durant et Pedone-Lauriel, 1867; BRINI, G.: Matrimonio e divorzio, op.cit., pp. 193-194; COSTA, E.: "Il concubinato a Roma», BIDR, XI (1898), p. 293 ss.; entre los autores que sostienen una teoría contraria y establecen que el concubinato era una relación de hecho tolerada por el Derecho, vid.: CASTELLI: «Il concubinato e la legislazione augustea», BIDR, XVII (1915), pp. 55 ss; Bonfante, P.: Corso..., op. cit., p. 316.

21. BONFANTE, P.: Corso..., op. cit., p. 316.

22. Ibid., pp. 316-317. 
obstante, al propagarse en todas las clases sociales y especialmente en las más elevadas, determinó que asumiera, algunas veces, el aspecto de un verdadero matrimonio morganático, pues incluso emperadores de alta moralidad como Vespasiano y Marco Aurelio, después de la muerte de su mujer, vivieron en concubinato y la concubina se consideró también indispensable para los gobernadores provinciales.

Una posición particular, casi jurídica, tuvo el concubinato de la liberta con su patrono ${ }^{23}$. Unión que no fue, en ningún momento, deshonrosa para la mujer, puesto que la concubina tomaba el nombre de matrona o materfamilias, denominación que siempre se había reservado para las mujeres legítimas del paterfami$\operatorname{lias}^{24}$. En época clásica se dejaba a voluntad del patrono mantener a su liberta como esposa o como concubina, aunque Ulpiano en D. 25, 7, 1, pr. consideraba más honroso para el patrón que conviviese en concubinato con su liberta.

D. 25, 7, 1, pr. - Ulpianus 2 ad l. iul. et pap.-Quae in concubinatu est, ab invito patrono poterit discedere et alteri se aut in matrimonium aut in concubinatum dare? ego quidem probo in concubina adimendum ei conubium, si patronum invitum deserat, quippe cum honestius sit patrono libertam concubinam quam matrem familias habere.

Por otra parte, este régimen especial determinaba que la concubina podía ser sometida a la acusación pública de adulterio, acción impensable fuera del matrimonium iustum, puesto que, evidentemente, no se podía acusar como marido a quien nunca fue su legítima mujer; no obstante, la acusación publica podía entablarse por un extraño, como expone Ulpiano en su libro II de adulteriis -iure tamen extranei accusationem instituere non prohibebitur, si modo ea sit, quae in concubinatum se dando matronae nomen non amisit, ut puta quae patroni concubina fuit- siempre que la mujer, aún uniéndose en concubinato, no hubiese perdido el titulo de matrona, y en efecto, la liberta que se unía en concubinato con su patrono no perdía su honestidad.

D. 48, 5, 14, pr. - Ulpianus 2 de adult.-Si uxor non fuerit in adulterio, concubina tamen fuit, iure quidem mariti accusare eam non poterit, quae uxor non fuit, iure tamen extranei accusationem instituere non prohibebitur, si modo ea sit, quae in concubinatum se dando matronae nomen non amisit, ut puta quae patroni concubina fuit.

Además, si la mujer había sido manumitida matrimonii causa no podía separarse de su patrón por voluntad propia, ni podía unirse en concubinato ni desposarse con otro hombre ${ }^{25}$.

Desde el punto de vista jurídico, la elevación del concubinato de relación social a institución jurídica se debió, sobre todo, al esfuerzo de los emperadores cristianos por abolirlo; hasta el punto, que llegaron a combatir estas parejas de hecho desde dos frentes: por un lado desprestigiando la condición de la concu-

23. CAstello, C.: In tema..., op. cit., pp. 76 ss.

24. Bonfante, P.: "Nota sulla riforma giustinianea del concubinato», Separata de Studi in onore di Silvio Perozzi, Palermo, 1924, p. 285.

25. D. 24, 2, 11 pr.- Ulpianus 3 ad l. iul. et pap. 
bina y de sus hijos y por otro, favoreciendo directamente la transformación del concubinato en matrimonio ${ }^{26}$.

Ambas vías fueron abiertas por Constantino. El emperador dictó tres constituciones sobre el particular ${ }^{27}$ y entre otras cuestiones desfavorables, decidió que no podían realizarse donaciones ni a la concubina ni a sus hijos aunque fuera de forma encubierta; si esto se hubiera cumplido, se les obligaba a restituir los bienes recibidos a los parientes consanguíneos del hombre cuando éste muriese -hijos legítimos, padres, hermanos-; condenándosele a pagar el cuádruplo, previa indagación bajo tortura, si no hubieran sido devueltos dichos bienes ${ }^{28}$.

Los emperadores posteriores, con oscilaciones, mitigaron o volvieron a aplicar el duro régimen de Constantino en su intento de combatir el concubinato.

Ahora bien, será Justiniano el que modifique el concepto del instituto y atenúe su régimen. En primer lugar, declaró abolida la categoría de mujeres con las cuales no se cometía stuprum de las leyes augusteas, +todas aquellas que no fuesen ingenuas y honestas+ y en segundo lugar, estableció que era jurídicamente lícito, previa declaración de los dos convivientes, el concubinato con mujer honesta e ingenua.

Una vez reconocido el concubinato como relación jurídica, se configuró como una unión conyugal inferior al matrimonio legítimo ${ }^{29}$, por lo que exigió las mismas condiciones establecidas para el matrimonio, que siguiendo a Bonfante $^{30}$ pueden señalarse las siguientes:

$1^{\circ}$.- El concubinato era rigurosamente monogámico, prohibiéndose al hombre que tenía esposa mantener una concubina y al soltero poseer más de una.

$2^{\circ}$.- Se requería para el concubinato la misma edad que para el matrimonio, esto es, que los convivientes fueran púberes.

$3^{\circ}$.- Estaba sujeto a los impedimentos de parentela consanguínea, por adopción o afinidad vigentes para el matrimonio.

Por otra parte, para calificar jurídicamente una unión conyugal como matrimonio o como concubinato se atendía exclusivamente a la voluntad inicial de las partes, esto es, que se hubiese querido constituir una u otra relación. No obstante, permaneció sin reconocimiento, el derecho de la concubina a participar en el grado de dignidad de su pareja. Ahora bien, se declaró abrogada la prohibición de donaciones a la concubina y a sus hijos.

Por último, Justiniano reguló definitivamente la legitimación de los liberi naturales, denominación que tomaban los hijos fruto del concubinato. La legitimación podía llevarse a cabo de tres formas distintas, primero, por el matrimonio posterior de sus padres - per subsequens matrimonium-; segundo, por concesión imperial -per rescriptum principis; tercero, por inscripción de los hijos en la curia del lugar donde tuviesen el domicilio sus padres -per oblationum curiae-.

26. BONFANTE, P.: Corso..., op. cit., p. 318.

27. C. TH. 4, 6, 2 y 3.

28. BONFANTE, P.: Corso..., op. cit., p. 318.

29. VOLTERRA, E.: Instituciones..., op. cit., p. 666.

30. Bonfante, P.: Corso..., op. cit., p. 324. 
Con la regulación del concubinato y sobre todo con la eliminación de los impedimentos para contraer matrimonio de las leyes augusteas, Justiniano logró, quizás, lo que no habían alcanzado los emperadores precedentes con sus severas sanciones, pues al suprimir la imposibilidad para contraer matrimonio con determinadas mujeres, consiguió que se fuera extinguiendo el concubinato.

El concubinato en Roma amparó uniones entre parejas de distinto sexo, que o no podían o no querían contraer matrimonio, pero nunca contempló la unión entre parejas homosexuales.

La institución fue abolida definitivamente en Oriente en tiempos de León el Filósofo ${ }^{31}$, estando vigente en Occidente hasta el siglo XII.

Hasta aquí he expuesto las uniones de hecho en Roma, pero antes de finalizar este trabajo deseo manifestar, como apunte final, que tanto el concepto de pareja de hecho como los requisitos exigidos en Roma para la constitución de las mismas, están presentes en la Ley 1/2001 que regula las uniones de hecho en la Comunidad Valenciana. No obstante, como puede contemplarse en su artículo primero, esta Ley es mucho más amplia y permisiva que el Derecho Romano, puesto que incluye en su ámbito de aplicación a las parejas homosexuales.

La Ley 1/2001 en su articulo primero dispone que para considerar una unión como pareja de hecho, ésta debe ser, libre, consentida, publica y notoria, estable, al menos durante un periodo ininterrumpido de doce meses y que exista una relación de afectividad. Por lo tanto, de igual manera que en Roma se excluyen las uniones casuales o de mera relación sexual y será la voluntad de los convivientes la que determine que esa pareja se constituya en unión de hecho.

Además, incidiendo en el consentimiento de las partes la Ley 1/2001 establece que para la aplicación de la ley, los convivientes deben someterse a la inscripción de la unión en el Registro Administrativo de Uniones de Hecho de la Comunidad Valenciana.

En cuanto a los requisitos personales que se encuentran enumerados en el artículo segundo de la citada Ley, podemos llegar a la conclusión de que existe paralelismo con los exigidos en el Derecho romano justinianeo para el concubinato o unión de hecho, pues, en primer lugar, en ambas regulaciones está presente el requisito de la edad, esto es, que para constituir una unión de hecho según las normas de la Ley 1/2001 ambos constituyentes deben ser mayores de edad o si son menores deben estar emancipados; en segundo lugar, se prohíbe la constitución de parejas de hecho a las personas ligadas por el vínculo de matrimonio o que hayan constituido una unión de hecho anterior que no haya sido disuelta y como ha podido comprobarse anteriormente, también los romanos prohibieron que el ciudadano casado mantuviese una concubina y que el soltero pudiese tener más de una; en tercer lugar, no se permite uniones de hecho entre los parientes por consanguinidad o adopción en línea recta y en línea colateral hasta el tercer grado, por lo tanto, la Ley se perfila rigurosamente monogámica y exogámica, aunque no incluya a los parientes afines.

31. Año 866-912 - siglo VII-VIII d.C. 
En cuanto a la disolución de las uniones de hecho, se encuentra similitud con las causas por las que se disolvía el matrimonio romano clásico y posteriormente el concubinato, al establecerse en el artículo sexto de la Ley 1/2001, que las uniones de hecho instituidas conforme a los dictados de la misma, se disuelven, bien por común acuerdo entre las partes o bien por decisión unilateral, muerte o matrimonio de uno de sus miembros. 